\title{
3D Antenna Array Design using Firefly Optimization Algorithm
}

\author{
Abdelmadjid RECIOUI", Youcef GRAINAT \\ Smart Grid Team, Laboratory of Signals and Systems, Institute of Electrical and Electronic \\ Engineering, University M'hamed Bougara of Boumerdes \\ Boulevard de l'indépendance, 35000, Boumerdes, Algeria \\ a_recioui@univ-boumerdes.dz
}

\begin{abstract}
The design of antenna arrays in a 3D geometry is presented in this Chapter. The decision variables considered for this synthesis problem are the array element amplitude excitations. The objective is to design an array which ensures minimum sidelobe level and a high directivity. The synthesis process is carried out using a nature-inspired global optimization technique. The optimization method based on the reaction of a firefly to the light of other fireflies is known as Firefly Algorithm (FA). It is a population-based iterative heuristic global optimization algorithm technique, developed by Xin-She Yang, for multi-dimensional and multi-modal problems. Simulation results for an antenna array with isotropic elements show that side lobe level is significantly reduced in non-uniform case. Besides, the directivity is not worse than that of the uniform one.
\end{abstract}

Keywords: 3D Antenna Arrays, Optimization, Firefly Algorithm, Sidelobe level, Directivity.

\section{INTRODUCTION}

In the 21 st century, thanks to large usage of mobile phones and modern technologies associated with them, a person now carries one or more antennas on them. This significant rate of growth is not likely to slow, as wireless communication systems become a larger part of everyday life. In addition, the strong growth in RFID (Radio Frequency Identity) devices suggests that the number of antennas in use would increase to one antenna per object in the world (products, containers, pets, bananas, toys, cds ... etc.). Hence, learning a little about antennas will contribute to one's overall understanding of the modern world [1].

Antennas are the basic components of any electric system and constitute the links between the transmitter and free space or free space and the receiver. They act as transducers that convert the current or voltage generated by the feeding circuit, such as a transmission line, a waveguide or coaxial cable, into an energy field propagating through space and vice versa. Thus, antennas play a very important role in

Cite this article as:

Abdelmadjid RECIOUI and Youcef GRAINAT, "3D Antenna Array Design using Firefly Optimization Algorithm", Algerian Journal of Signals and Systems, Vol. 4, Issue 2, December 2019.pp: 61-70. describing the characteristics of the system in which antennas are employed [2-4].

Antennas are employed by various systems in different forms. For wireless communication systems, the antenna is one of the most critical components. A good design of the antenna can relax system requirements and improve the overall system performance. So, to meet the particular need at hand, usually antennas are required to optimize the radiation energy in some directions and suppress it in others. They must then take various forms. They may be a piece of conducting wire, an aperture, a patch, an assembly of elements (array), a reflector, a lens, and so forth. In long distance communication, there is great need for very directive antennas with very high gain [1-8].

Due to the radiation pattern limitations of a single antenna, several single antenna elements can be combined to form an array. The technology based in antenna arrays is a key solution for current and future wireless communication systems. Therefore, several antenna array geometries have been developed to meet the special needs, among others, of the applications such as: beam steering, directivity (DIR), gain, angular coverage, side lobe level (SLL) [9-11]. However, there exist applications where the traditional antenna arrays cannot achieve the objectives such as tracking, direction finding 
and satellites communications. Under these conditions, 3D geometric antenna arrays have raised as an attractive solution. Research reports concerning antenna array designs for 3D geometries are increasingly receiving attention for their capabilities for forming different radiation shapes and for scanning over all angles [12-15]. Yet, a synthesis of the pattern needs to be performed to achieve the maximum performance of the antenna system. Theoretically, the array should be designed with a maximum directivity and minimum side lobe level so as to achieve maximum signal to noise plus interference ratio at the output of the array antenna. However, these two antenna properties turn out to be conflicting meaning that designing to enhance one of them would affect the other and vice versa. Hence, a trade-off must be achieved where optimization takes part in the design process [1-11].

Due to the amazing development of computers, applications of numerical optimization techniques to antenna design have become possible. Among these techniques, bio-inspired algorithms like the firefly algorithm (FA) have been found to be effective in optimizing difficult multidimensional problems in a variety of fields [16]. In the last two decades, more than a dozen of new metaheuristic algorithms such as particle swarm optimization [17], differential evolution [12], biogeography based algorithm [18], firefly algorithm [19] and Taguchi search method [20] have appeared and they have shown great potential in solving tough engineering optimization problems. Among these new algorithms, it has been shown that firefly algorithm is very efficient in dealing with multimodal, global optimization problems. Firefly algorithm (FA) is a new populationbased metaheuristic approach developed by Xin-She Yang, which is nature-inspired by behavior of the flashing characteristics of fireflies. In this context, the flashing light can be formulated in such a way that it is associated with the objective function to be optimized, which makes it possible to formulate the firefly algorithm [21-24].

In this paper, Focus is put on optimizing directivity and side-lobe levels of uniformly spaced antenna elements along a cubic geometry by varying the element excitation amplitudes using firefly algorithm optimization.

\section{PROBLEM MATHEMATICAL FORMULATION}

A 3D array antenna, see Figure 1 , is achieved by introducing a number of planar arrays on the $\mathrm{z}$ axis. In this case, the array factor of $\mathrm{N}$ by $\mathrm{M}$ by $\mathrm{H}$ uniform array is:

$$
=\frac{\sin \left(\frac{N \Psi_{x}}{2}\right) \sin \left(\frac{M \Psi_{y}}{2}\right) \sin \left(\frac{H \Psi_{z}}{2}\right)}{N \sin \left(\frac{\Psi_{x}}{2}\right) M \sin \left(\frac{\Psi_{y}}{2}\right) H \sin \left(\frac{\Psi_{z}}{2}\right)}
$$$$
\text { Where }
$$$$
\Psi_{\mathrm{x}} \alpha_{\mathrm{x}} \mathrm{kd}_{\mathrm{x}} \sin \theta \cos \varphi
$$$$
\Psi_{\mathrm{y}}=\mathrm{a}_{\mathrm{y}}+\mathrm{kd}_{\mathrm{y}} \sin \theta \sin \varphi
$$$$
\Psi_{\mathrm{z}}=\mathrm{a}_{\mathrm{z}}+\mathrm{kd}_{\mathrm{z}} \cos \theta
$$

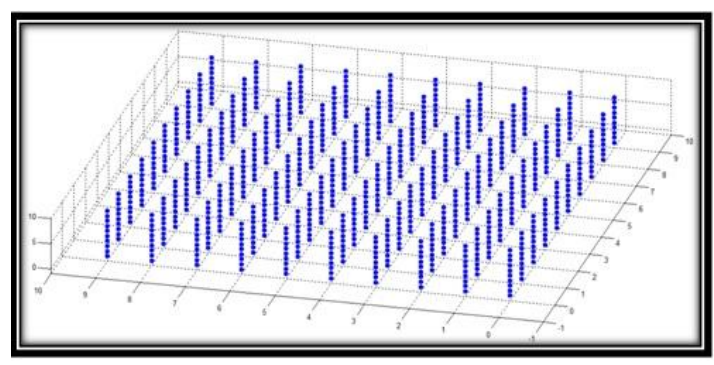

Figure 1 3D-Cubic antenna array

For an arbitrary N-element 3D antenna array, the radiation pattern is given as [13]:

$P(\theta, \varphi)=f(\theta, \varphi) A F(\theta, \varphi)$

Where $\mathrm{f}(\theta, \varphi)$ denotes the element pattern. $\operatorname{AF}(\theta, \varphi)$ is the array factor and it is given as[14]:

$\operatorname{AF}(\theta, \varphi)=\sum_{n=1}^{N} I_{n} e^{j \alpha_{n}+j k \hat{r} \cdot r}$

After some mathematical manipulations, the array factor can be written as [13-14]:

$\operatorname{AF}(\theta, \varphi)$

$=\sum_{n=1}^{N} I_{n} e^{j\left[k\left(x_{n} \sin \theta \cos \varphi+y_{n} \sin \theta \sin \varphi+z_{n} \cos \theta\right)+\alpha_{n}\right]}$

Equation 5 represents the multiplication principle that says: "the array pattern can be considered to be the product of the element pattern and the array factor. The array factor depends only on the position of each element and amplitude and phase of the exciting current."

In the following sections, it is assumed that the radiating elements of the array are isotropic, thus $\mathrm{f}(\theta, \varphi)=1$, and the array pattern is determined completely by the array 
factor $\operatorname{AF}(\theta, \varphi)$. In fact, the elements being isotropic and point sources allow us to ignore any mutual coupling between the elements and the element excitation is solely its fed excitation current.

In the uniform case, the excitation amplitude $\mathrm{I}=1$ for all elements. For the non-uniform case, the excitation amplitude (I) will be varied in the interval[0 1]. To achieve the design objectives, two objective functions to be minimized are considered. The first is merely the sidelobe level only. The second is the sidelobe level and the directivity optimized at the same time. Explicitly, the two functions are written as:

$f_{1}=S L L_{\text {max }}(I)$

$=20 \log _{10}\left|\frac{A F(\theta)}{A F(\theta)_{\max }}\right|$ in the side lobe region

And

$\mathrm{f}_{2}=\mathrm{f}_{3}+10 \mathrm{f}_{1}$

With

$\mathrm{f}_{3}=-\operatorname{DIR}(\mathrm{I})$

$=-10 \log _{10}\left(\frac{4 \pi \max \left(\max \left(\mid \mathrm{AF}^{2}\right)\right)}{\left.\sum \sum \mathrm{AF}\right|^{2} \sin (\theta) \mathrm{d} \theta \mathrm{d} \varphi}\right)$

Where:

$A F$ : is the array factor of the cubic antenna array

SLLmax: is the maximum side lobe level in $\mathrm{dB}$

$D I R$ : is the directivity of the cubic antenna array in $\mathrm{dB} \cdot \mathrm{d} \theta=\frac{\pi}{\mathrm{A}}$ and $\mathrm{d} \varphi=\frac{2 \pi}{\mathrm{B}}$ with $\mathrm{A}$ and $\mathrm{B}$ being positive real numbers that define the needed resolution. The antenna array considered here is a uniformly spaced $\left(\mathrm{d}_{\mathrm{x}}=\mathrm{d}_{\mathrm{y}}=\mathrm{d}_{\mathrm{z}}\right)$ cubic antenna array of 10 by 10 by 10 elements.

\section{THE FIREFLY ALGORITHM}

Optimization is one of the basic mathematical techniques that lie at the heart of modern competitive management, design and development. It is the act of achieving the best possible result under given circumstances. In design, construction, maintenance...etc., engineers have to take many technological and managerial decisions at several stages [25].

The main objective of the optimization methods is to determine the maximum or the minimum of mathematical functions, called objective functions, which may be, or may not be, subject to constraints on its variables.
Due to the wide variety of practical applications, optimization algorithms have been increasingly studied in the area of engineering and applied mathematics. The optimization methods can be divided into two major groups: deterministic and stochastic methods, which may use or not the derivatives of the objective and constraint functions.

The most of deterministic methods are local search methods. These methods are characterized for producing always the same set of solutions (optimal points) if the algorithm start under the same initial conditions. In turn, the stochastic methods are characterized by having one or more components of randomness, called stochastic components. This implies that for the same problem, and subject to the same initial conditions, these algorithms may not generate the same optimal solutions [26].

There is a range of possibilities of how to form this stochastic component. For example, one way is to make a simple randomization by randomly sampling the search space or by making random walks. The majority of this type of methods is considered as metaheuristic [27].

Firefly Algorithm (FA) is an algorithm that belongs to the second group, that is, a stochastic and metaheuristic algorithm, and it was developed by Yang. It is a recent nature inspired optimization algorithm, inspired by the social behavior of fireflies, and is based on their flashing and attraction characteristics. [16]

Firefly Algorithm (FA) is one of the recent swarm intelligence methods developed by Xin-She Yang in 2008. FA is a stochastic, nature-inspired, metaheuristic algorithm that can be applied for solving the hardest optimization problems [16].

\subsection{Overview of Firefly Algorithm}

FA is an optimization algorithm inspired by behavior and motion of fireflies. It is a population-based optimization algorithm which uses swarm intelligence to converge. It is similar to other optimization algorithms employing swarm intelligence such as PSO, $G A$ and DEA. But $F A$ is found to have superior performance in many cases. [22]

FA initially produces a swarm of fireflies located randomly in the search space. The initial distribution is usually produced from a uniform random distribution. The position of 
each firefly in the search space represents a potential solution of the optimization problem. The dimension of the search space is equal to the number of optimizing parameters in the given problem.

The fitness function takes the position of a firefly as input and produces a single numerical output value denoting how good the potential solution is. A fitness value is assigned to each firefly.

The FA uses a phenomenon known as bioluminescent communication to model the movement of the fireflies through the search space. [22] The velocity or the pull of a firefly towards another firefly depends on the attractiveness. The attractiveness depends on the relative distance between the fireflies. It is a function of the brightness of the fireflies as well. A brighter firefly that is far away may not be as attractive as a less bright firefly that is closer. In each iterative step, FA computes the brightness and the relative attractiveness of each firefly. Depending on these values, the positions of the fireflies are updated. After a sufficient amount of iterations, all fireflies converge to the best possible position on the search space. [28]

The firefly algorithm is based on three main principles [16]:

1. All fireflies are unisex, implying that all the elements of a population can attract each other.

\begin{tabular}{|l|}
\hline Firefly Algorithm \\
Objective function $f(\mathbf{x}), \quad \mathbf{x}=\left(x_{1}, \ldots, x_{d}\right)^{T}$ \\
Initialize a population of fireflies $\mathbf{x}_{i}(i=1,2, \ldots, n)$ \\
Define light absorption coefficient $\gamma$ \\
while $(t<$ MaxGeneration) \\
for $i=1: n$ all $n$ fireflies \\
for $j=1: i$ all $n$ fireflies \\
Light intensity $I_{i}$ at $\mathbf{x}_{i}$ is determined by $f\left(\mathbf{x}_{i}\right)$ \\
if $\left(I_{j}>I_{i}\right)$ \\
$\quad$ Move firefly $i$ towards $j$ in all $d$ dimensions \\
$\quad$ end if \\
$\quad$ Attractiveness varies with distance $r$ via exp $[-\gamma r]$ \\
$\quad$ Evaluate new solutions and update light intensity \\
end for $j$ \\
end for $i$ \\
Rank the fireflies and find the current best \\
end while \\
Postprocess results and visualization \\
\hline
\end{tabular}

Figure 2 Pseudo code of the firefly algorithm (FA) [23]

2. The attractiveness between fireflies is proportional to their brightness. The firefly with less bright will move towards the brighter one. If no one is brighter than a particular firefly, it moves randomly. Attractiveness is proportional to the brightness which decreases with increasing distance between fireflies.

3. The brightness or light intensity of a firefly is related with the type of function to be optimized. In practice, the brightness of each firefly can be directly proportional to the value of the objective function.

Based on these three rules, the basic steps of the firefly algorithm (FA) can be summarized as the pseudo code shown in Figure 2.

\subsection{Attractiveness}

In the FA, there are two important issues: the variation of light intensity and formulation of the attractiveness. For simplicity, we can always assume that the attractiveness of a firefly is determined by its brightness or light intensity which in turn is associated with the encoded objective function.

In the simplest case for maximum optimization problems, the brightness I of a firefly at a particular location $x$ can be chosen as $\mathrm{I}(\mathrm{x}) \propto \mathrm{f}(\mathrm{x})$. However, the attractiveness $\beta$ is relative. It should be seen in the eyes of the beholder or judged by the other fireflies. Thus, it should vary with the distance rij between firefly i and firefly j. As light intensity decreases with the distance from its source, and light is also absorbed in the media, we should allow the attractiveness to vary with the degree of absorption.

In the simplest form, the light intensity I(r) varies according to the inverse square $\operatorname{lawI}(r)=\frac{I_{s}}{r^{2}}$, where $I_{s}$ is the intensity at the source.

For a given medium with a fixed light absorption coefficient $\mathrm{Y}$, the light intensity (I) varies with the distance $r$. that is:

$$
\mathrm{I}=\mathrm{I}_{0} \mathrm{e}^{-\mathrm{rr}}
$$

Where:

$\mathrm{I}_{0}=$ is the original light intensity.

In order to avoid the singularity at $r=0$ in the expressionI $(r)=\frac{I_{s}}{r^{2}}$, the combined effect of both the inverse square law and absorption can be approximated using the following Gaussian form:

$\mathrm{I}(\mathrm{r})=\mathrm{I}_{0} \mathrm{e}^{-\gamma \mathrm{r}^{2}}$

Sometimes, we may need a function which decreases monotonically at a slower 
rate. In this case, we can use the following approximation:

$$
\mathrm{I}(\mathrm{r})=\frac{\mathrm{I}_{0}}{1+\mathrm{Vr}^{2}}
$$

At a shorter distance, the above two forms are essentially the same. This is because the series expansions about $r=0$ are equivalent to each other up to the order of $\mathrm{O}(\mathrm{r} 3)$.

$$
\begin{aligned}
& \mathrm{e}^{-\gamma \mathrm{r}^{2}} \approx 1-\mathrm{yr}^{2}+\frac{1}{2} \mathrm{y}^{2} \mathrm{r}^{4}+ \\
& \frac{1}{1+\mathrm{rr}^{2}} \approx 1-\mathrm{yr}^{2}+\mathrm{y}^{2} \mathrm{r}^{4}+\cdots
\end{aligned}
$$

As a firefly's attractiveness is proportional to the light intensity seen by adjacent fireflies, we can now define the attractiveness $\beta$ of a firefly by:

$\beta(r)=\beta_{0} e^{-\gamma r^{2}}$

Where $\beta_{0}$ is the attractiveness at $r=0$.

As it is often faster to calculate $\frac{1}{\left(1+r^{2}\right)}$ than an exponential function, the above function, if necessary, can conveniently be replaced by:

$\beta(r)=\frac{\beta_{0}}{1+\gamma r^{2}}$

The above Equation defines a characteristic distance:

$\Gamma=\frac{1}{\sqrt{\mathrm{Y}}}$

Over which the attractiveness changes significantly from $\beta_{0}$ to $\beta_{0} \mathrm{e}^{-1}$.

In the implementation, the actual form of attractiveness function $\beta(r)$ can be any monotonically decreasing functions such as the following generalized form:

$\beta(r)=\beta_{0} \mathrm{e}^{-\mathrm{\gamma r}^{\mathrm{m}}}, \quad(\mathrm{m} \geq 1)$

For a fixed $Y$, the characteristic length becomes:

$\Gamma=\mathrm{y}^{-1 / \mathrm{m}} \rightarrow 1$ as $\mathrm{m} \rightarrow \infty$

Conversely, for a given length scale in an optimization problem, the parameter $y$ can be used as a typical initial value. That is:

$\mathrm{Y}=\frac{1}{\Gamma^{\mathrm{m}}}$

\subsection{Distance and Movement}

The distance between any two fireflies $\mathrm{i}$ and $j$ at $x i$ and $x j$, respectively, is the Euclidean distance:

$\mathrm{r}_{\mathrm{ij}}=\left\|\mathrm{x}_{\mathrm{i}}-\mathrm{x}_{\mathrm{j}}\right\|=\sqrt{\sum_{\mathrm{k}=1}^{\mathrm{d}}\left(\mathrm{x}_{\mathrm{i}, \mathrm{k}}-\mathrm{x}_{\mathrm{j}, \mathrm{k}}\right)^{2}}$

Wherex $_{\mathrm{i}, \mathrm{k}}$ : is the kth component of the spatial coordinate $x i$ of ith firefly.
The movement of a firefly $i$ is attracted to another more attractive (brighter) firefly $\mathrm{j}$ is determined by:

$x_{i}=x_{i}+\beta_{0} e^{-\gamma r_{i j}^{2}}\left(x_{j}-x_{i}\right)+\alpha\left(\right.$ rand $\left.-\frac{1}{2}\right)(23)$ where the second term is due to the attraction while the third term is randomization with $\alpha$ being the randomization parameter, rand is a random number generator uniformly distributed in $[0,1]$.For most cases we can take $\beta_{0}=1$ and $\alpha \in[0$, $1]$.

Furthermore, the randomization term can easily be extended to a normal distribution $\mathrm{N}$ $(0,1)$ or other distributions.

\subsection{FA Summarized}

The important steps of the FA are summarized below [24]:

1. Initialization: The number of fireflies in the population space is $\mathrm{K}$. The position of the nth firefly is denoted by a vector $\mathrm{Wn}$, where each measurement indicates the weight of an array element.

$\mathrm{W}_{\mathrm{n}}=\left(\mathrm{w}_{\mathrm{n}}^{1}, \mathrm{w}_{\mathrm{n}}^{2}, \mathrm{w}_{\mathrm{n}}^{3}, \cdots \mathrm{w}_{\mathrm{n}}^{\mathrm{t}}, \mathrm{w}_{\mathrm{n}}^{\mathrm{N}}\right)$

Where $n=1,2,3 \ldots K$ and $t=1,2,3 \ldots N$. To initialize the location of $\mathrm{K}$ fireflies in $\mathrm{N}$ dimensional search space, which are randomly selected with in the search boundary as:

$\mathrm{w}_{\mathrm{n}}^{\mathrm{t}}=\mathrm{w}_{\mathrm{L}}^{\mathrm{t}}+\left(\mathrm{w}_{\mathrm{H}}^{\mathrm{t}}-\mathrm{w}_{\mathrm{L}}^{\mathrm{t}}\right) \times \operatorname{rand}()$

Where $\mathrm{w}_{\mathrm{L}}^{\mathrm{t}}$ and $\mathrm{w}_{\mathrm{H}}^{\mathrm{t}}$ represents the lower and upper values of the th variable in the population respectively, and $\operatorname{rand}()$ is a uniform random variable with values ranging from 0 to 1 .

2. Evaluation: Calculate the fitness for each firefly position in the population and sort the population from brightest to lightest. The brightness of each firefly is calculated at current generation by the fitness function at their current location. The brightest or light intensity is inversely proportional to cost function of individual firefly for minimization problem. 
3. Update the Location of Fireflies: Depending on the attractiveness, each firefly in the population will move toward the adjacent firefly with more light intensity and its position is updated for the next generation. The firefly i (less intensity) will move toward the other fireflies $\mathrm{j}$ that are brighter. There are two important issues in the FA, the deviation of brightness or light intensity and formation of the attractiveness. The attractiveness of a firefly is calculated by its brightness or light intensity which is directly associated with the cost function. The brightness of the nth firefly $\mathrm{Bn}$ is given by the equation:

$\mathrm{B}_{\mathrm{n}}=\mathrm{f}_{\text {fitness }}\left(\mathrm{w}_{\mathrm{n}}\right)$

The attractiveness between the i-th and j-th firefly is given by:

$\beta_{\mathrm{ij}}=\beta_{0} \mathrm{e}^{-\mathrm{\gamma r}_{\mathrm{ij}}{ }^{2}}$

Where:

$\beta$ : is a constant whose value is 1 .

$\mathrm{Y}$ : is dynamic range of search space.

$r_{i j}$ : is a distance between wi and wj given by the equation:

$r_{i j}=\left\|w_{j}-w_{i}\right\|=\sqrt{\sum_{n=1}^{N}\left(w_{j}^{t}-w_{i}^{t}\right)^{2}}$

The position of firefly is updated in each iterative step. If the intensity/brightness of $j$-th firefly is greater than the brightness of the i-th firefly, then the i-th firefly moves towards the $\mathrm{j}$-th firefly. The motion of the fireflies is denoted by the following equation:

$w_{n}=w_{n}+\beta_{i j}\left(w_{j}-w_{i}\right)+\alpha \varepsilon_{n}$

Where: $\alpha$ is a constant parameter of scale whose value depends on the dynamic range of the solution space. The third term is randomization with the vector of random variables $\varepsilon_{\mathrm{n}}$ being drawn from a Gaussian distribution [23].

4. Ranking and Computation of Global Best: On the basis of their brightness, the fireflies are ranked in the current generation, and the brightness of each firefly is compared with all other fireflies, and the location of the brightest firefly in the population is taken as current global best, and, in this way, for the brightest firefly we received a best fitness value in the recent generation.

5. Termination of Program: When fitness function achieves a certain prescribed value, or when maximum number of cycles (NOC) is reached, the program terminates and stores the best value, otherwise it goes back to step 2 to 4 . The location of the best firefly gives the optimum solution and the corresponding brightness of the firefly that gives the optimum fitness value of the fitness function. The flowchart of FA is shown in Figure 3.

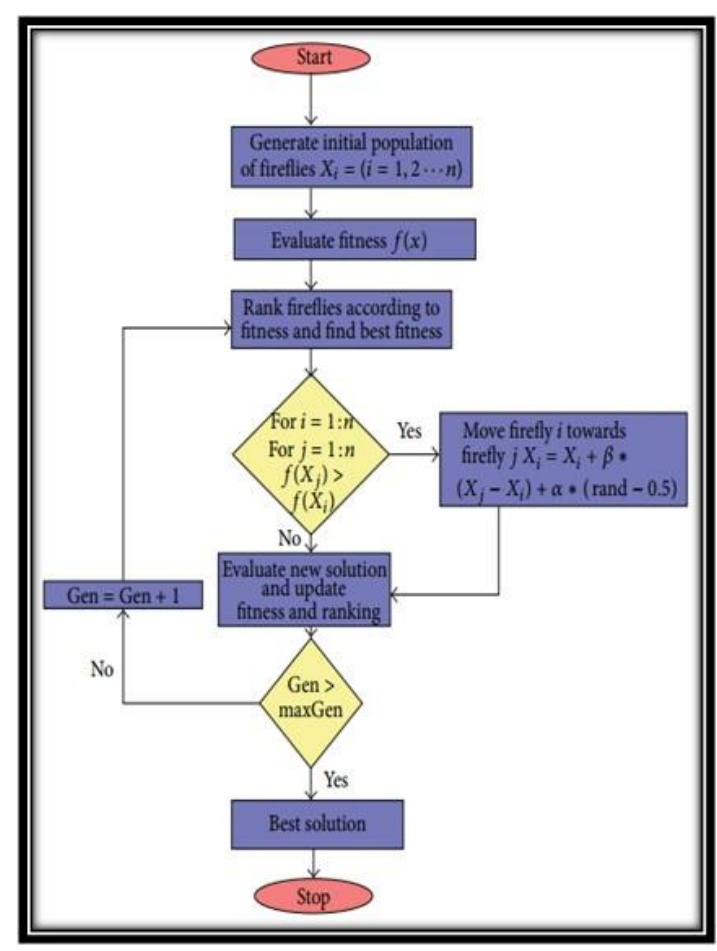

Figure 3 The flowchart of Firefly Algorithm

\section{RESULTS AND DISCUSSIONS}

The array factor of array antennas is presented in equation (7). For 10 by 10 by 10 cubic antenna array, the array factor is:

$\operatorname{AF}(\theta, \varphi)$

$=\sum_{\substack{\mathrm{h}=1 \\ \text { Where: }}}^{\mathrm{H}} \sum_{\mathrm{m}=1}^{\mathrm{N}} \mathrm{I}_{\mathrm{m}, \mathrm{n}, \mathrm{h}} \mathrm{e}^{\mathrm{j}\left[(\mathrm{h}-1) \Psi_{\mathrm{z}}+(\mathrm{n}-1) \psi_{\mathrm{y}}+(\mathrm{m}-1) \psi_{\mathrm{x}}\right]}$

$I_{m, n, h}$ : is the excitation current of the elements.

$\Psi_{\mathrm{x}}=\mathrm{kd}_{\mathrm{x}} \sin \theta \cos \varphi+\mathrm{a}_{\mathrm{x}}$ 
$\Psi_{\mathrm{y}}=\mathrm{kd}_{\mathrm{y}} \sin \theta \sin \varphi+\alpha_{\mathrm{y}}$

$\Psi_{\mathrm{z}}=\mathrm{kd}_{\mathrm{z}} \cos \theta+\mathrm{a}_{\mathrm{z}}$

$\mathrm{a}_{\mathrm{x}}=-\mathrm{kd}_{\mathrm{x}} \sin \theta_{\mathrm{s}} \cos \varphi_{\mathrm{s}}$

$\mathrm{a}_{\mathrm{y}}=-\mathrm{kd}_{\mathrm{y}} \sin \theta_{\mathrm{s}} \sin \varphi_{\mathrm{s}}$

$\mathrm{a}_{\mathrm{z}}=-\mathrm{kd}_{\mathrm{z}} \cos \theta_{\mathrm{s}}$

$\mathrm{k}=\frac{2 \pi}{\lambda}$

We have:

$$
\begin{gathered}
M=N=H=10 \\
d_{x}=d_{y}=d_{z}=\frac{\lambda}{2} \\
\theta_{s}=\varphi_{s}=45^{\circ}
\end{gathered}
$$

Fo the firefly algorithm, 50 fireflies with 1000 iterations are considered with values of $\alpha=0.5, \beta=0.2$, and $\gamma=1$.

\subsection{The uniform case}

The relative array factor of the uniform case is shown in Figure 4 and Figure 5 for $\varphi=0^{\circ}$ and $\varphi=90^{\circ}$, respectively. The array factor is characterized by a directivity of $25.3567 \mathrm{~dB}$ and a sidelobe level of $-8.0139 \mathrm{~dB}$ when $\varphi=0^{\circ}$, and a sidelobe level of $-6.8876 \mathrm{~dB}$ along $\varphi=90^{\circ}$.

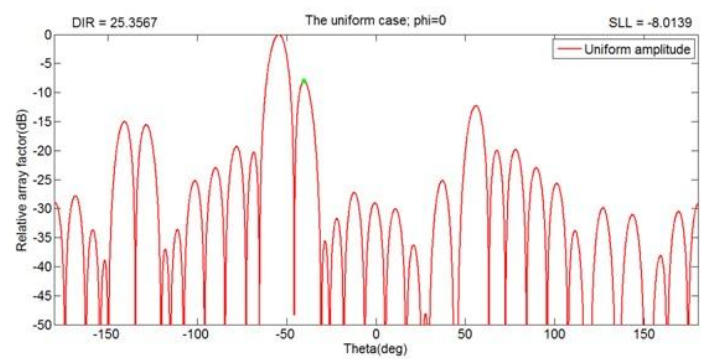

Figure 4 The array factor for the uniform case; phi $=0^{\circ}$

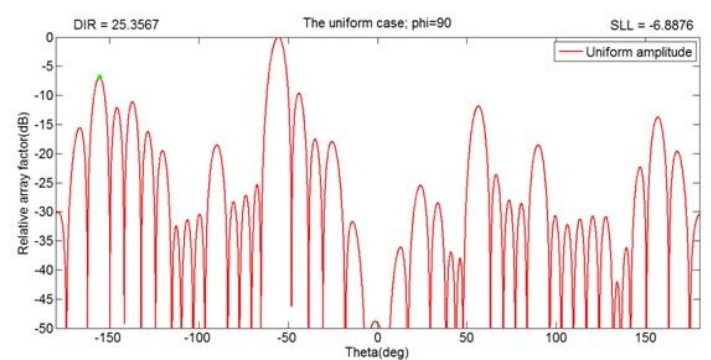

Figure 5 The array factor for the uniform case; phi $=90^{\circ}$

\subsection{The non-uniform case: Amplitude Variation}

\subsubsection{Optimization of the Side Lobe Level only}

By setting the objective function to $f_{1}$, which is presented in the equation (8), and varying the amplitudes of the excitation current of the elements in the interval [ $\left[\begin{array}{ll}0 & 1\end{array}\right]$, the optimized array factor along $\varphi=0^{\circ}$ is shown in Figure 6 . The side lobe level is improved from $8.0139 \mathrm{~dB}$ to $-37.371 \mathrm{~dB}$. However, the directivity has been reduced from $25.3567 \mathrm{~dB}$ to $25.14 \mathrm{~dB}$. Note that the loss in directivity is not that worse compared to the gain in sidelobe level.

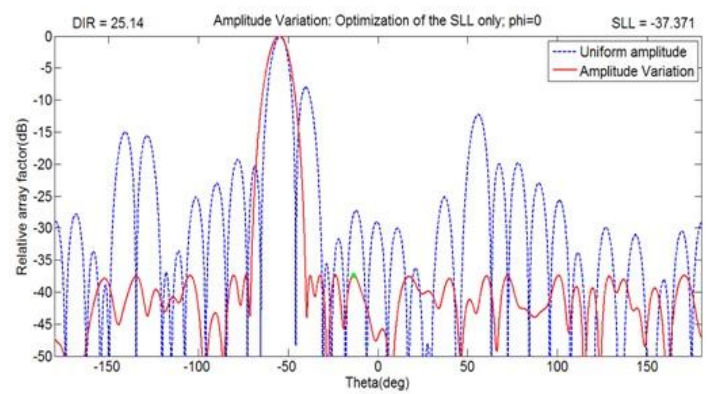

Figure 6 Amplitude Variation for SLL Only; phi $=0^{\circ}$

b- $\varphi=90^{\circ}$

After optimization along $\varphi=90^{\circ}$, the produced array factor is shown in Figure 7. The optimization using FA has been able to reduce the sidelobe level from a value of $6.8876 \mathrm{~dB}$ to $-31.8464 \mathrm{~dB}$ which is also a better value. Note again that the directivity has been reduced from 25.3567dBto $25.0852 \mathrm{~dB}$. This decrease is again not much noticeable compared to the gain in sidelobe level reduction.

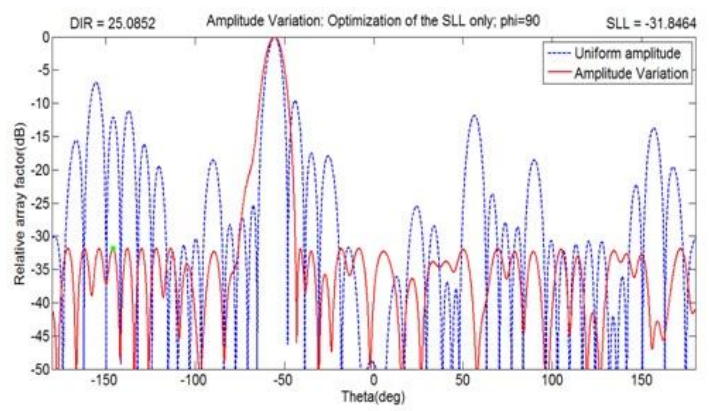

Figure 7 Amplitude Variation for SLL Only; $\mathrm{phi}=90^{\circ}$

\subsubsection{Optimization of the Side Lobe Level and the Directivity}

a- $\varphi=0^{\circ}$

a- For $\varphi=0$ 
By setting the objective function to $\mathrm{f}_{2}$, which is presented in the equation (9), and varying the amplitudes of the excitation current of the elements in the interval [0 1$]$, the optimized relative array factor is given in Figure 8.

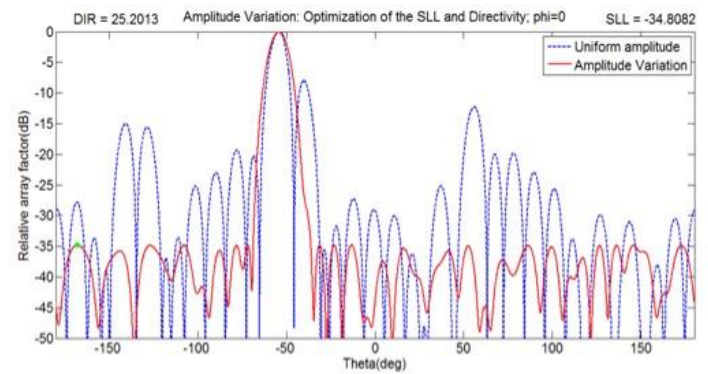

Figure 8 Amplitude Variation for both SLL and Directivity; $\mathrm{phi}=0^{\circ}$

The sidelobe level has been reduced from $8.0139 \mathrm{~dB}$ to $-34.8082 \mathrm{~dB}$. Once more, the directivity has been reduced from $25.3567 \mathrm{~dB}$ to $25.2013 \mathrm{~dB}$. Notice, however, that compared to the first case, the compromise between the directivity and sidelobe level is better for this case.

\section{b- $\varphi=90$}

By setting the objective function to $f_{3}$ again, and varying the amplitudes of the excitation current of the elements in the interval $\left[\begin{array}{ll}0 & 1\end{array}\right]$, we got the relative array factor that is shown in Figure 9. There has been a reduction in side lobe level from $6.8876 \mathrm{~dB}$ to $-31.7007 \mathrm{~dB}$. Once more, the directivity has been reduced from $25.3567 \mathrm{~dB}$ to $25.072 \mathrm{~dB}$. Again, the trade-off between the sidelobe level and directivity is better for this case than the sidelobe level only case.

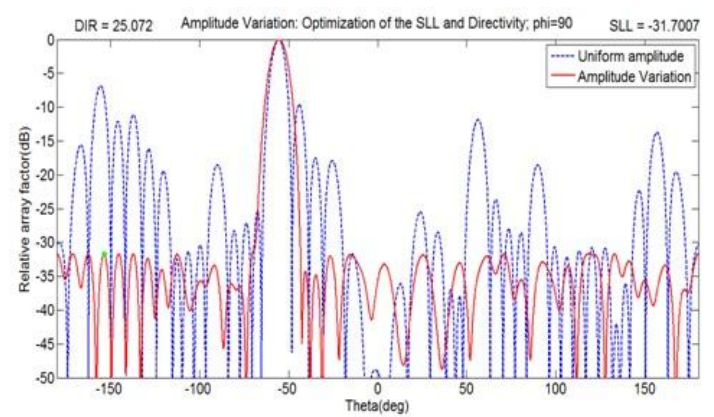

Figure 9 Amplitude Variation: SLL and Directivity; phi $=90$

\subsubsection{Summary and Discussions}

In this paper, we have optimized the SLL and the directivity of a 10 by 10 by 10 Cubic antenna array using firefly algorithm by varying the amplitude of the excitation current of the elements, and the results are summarized in Table 1. One can see that the Firefly algorithm has reached satisfying values of sidelobe levels in the amplitude variation part, with an acceptable slight reduction in directivity values.

Table 1 Summary of the FA optimization results

\begin{tabular}{|c|c|c|c|c|}
\hline \multirow[b]{2}{*}{ The case } & & \\
\hline & $\begin{array}{l}\text { Parameters to be } \\
\text { optimized for }\end{array}$ & $\varphi$ & SLL & DIR \\
\hline \multirow{2}{*}{$\begin{array}{l}\text { Uniform } \\
\text { case }\end{array}$} & \multirow{6}{*}{$\begin{array}{c}\text { Side Lo be Level only } \\
\text { Side Lo be Level and } \\
\text { Directivity }\end{array}$} & $\varphi=0^{\circ}$ & $-8.0139 \mathrm{~dB}$ & $25,3567 \mathrm{~dB}$ \\
\hline & & $\varphi=90^{\circ}$ & $-6.8876 \mathrm{~dB}$ & $253567 \mathrm{~dB}$ \\
\hline \multirow{4}{*}{$\begin{array}{l}\text { Non- } \\
\text { Uniform } \\
\text { case }\end{array}$} & & $\varphi=0^{\circ}$ & $-37.371 \mathrm{~dB}$ & $25,1400 \mathrm{~dB}$ \\
\hline & & $\varphi=90^{\circ}$ & $-31,8464 \mathrm{~dB}$ & $25,0852 \mathrm{~dB}$ \\
\hline & & $\varphi=0^{\circ}$ & $-348082 \mathrm{~dB}$ & $25,2013 \mathrm{~dB}$ \\
\hline & & $\varphi=90^{\circ}$ & $-31,7007 \mathrm{~dB}$ & $25.0720 \mathrm{~dB}$ \\
\hline
\end{tabular}

\section{CONCLUSION}

In this chapter, the recently developed biologically- inspired meta-heuristic algorithm called firefly algorithm (FA) has been used to solve3D antenna array design problem. The design problem has been formulated as an optimization task to achieve sidelobe level reduction by varying the element excitation amplitudes.

The FA algorithm was able to generate the non-uniform excitation amplitudes for the cubic arrays in question. The performance of the antenna arrays was improved in term of side lobe level and directivity. The simulated results reveal that the optimal design offers a considerable SLL reduction along with reduction of Directivity compared to the corresponding uniform array. The FA algorithm has successfully obtained the minimum SLL and a close value of the uniform directivity.

For future research, FA can be exploited with focus on other characteristics such as gain, beamwidth, and null control by varying more parameter like element spacing and phase shift. It can also be tried with practical elements like dipole or microstrip antennas. In this case, more characteristics can be explored including mutual coupling and VSWR.

Overall, the firefly algorithm has proved to be a very useful tool finding the best solutions in the analysis and design of antenna systems. 


\section{Acknowledgments}

This work is part of the research activities within the Laboratory of Signals and Systems, university of Boumerdes, Algeria. Special thanks to Professor Hamid BENTARZI, the director of the signals and systems Laboratory at the University of Boumerdes-Algeria for his valuable advice and continual support.

\section{REFERENCES}

[1] Gupta Sumit Kumar, Jangam Harish Kumar, Sharma Nipun. Theory of Antennas, Its Advantage \& Applications in Communication Systems. International Journal of Engineering Development and Research (IJEDR). 2018;6(1):925-930.

[2] Recioui Abdelmadjid. Optimization of Antenna Arrays Using Different Strategies Based on Taguchi Method. Arabian Journal for Science and Engineering.2014;39(2):935-944. https://doi.org/10.1007/s13369-013-0644-8...

[3] Recioui Abdelmadjid, Bentarzi Hamid. Capacity Optimization of MIMO Wireless Communication Systems Using a Hybrid Genetic-Taguchi Algorithm. Wireless Personal Communications. 2013; 71(2):1003-1019. DOI: https://doi.org/10.1007/s11277-012-0857-2.

[4] Balanis Constantine A. Antenna Theory: Analysis and Design ( $3^{\text {rd }}$ edition). John wiley and sons Inc. 2005.

[5] Recioui Abdelmadjid. Application of a GalaxyBased Search Algorithm to MIMO System Capacity Optimization. Arabian Journal for Science and Engineering. 2015; 41: 3407. DOI: 10.1007/s13369-015-1934-0.

[6] Recioui Abdelmadjid. Design and Thinning of Linear and Planar Antenna Arrays Using a Binary Teaching Learning Optimizer. ACTA PHYSICA

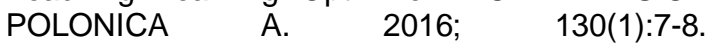
DOI:10.12693/APhysPolA.130.7

[7] Recioui Abdelmadjid, Benabid Mondher, Djilani Nabil. Rectangular Antenna Array Optimization using Wind Driven Optimization. Algerian Journal of Signals and Systems. 2016;1(2):109-120.

[8] A. Recioui. Application of the Spiral Optimization Technique to Antenna Array Design. In: Pandian Vasant, Sirma Zeynep AlparslanGok, Gerhard-Wilhelm Weber.Handbook of Research on Emergent Applications of Optimization Algorithms, IGI Global. 2018. p812. DOI: 10.4018/978-1-5225-2990-3.ch018.

[9] Khodier M. M., Christodoulou C. G. Linear array geometry synthesis with minimum side lobe level and null control using particle swarm optimization. IEEE Transactions on Antennas and Propagation.2005; 53(8): 2674-2679. DOI:10.1109/TAP.2005.851762

[10] Lin C. , Qing A. , Feng Q. Synthesis of unequally spaced antenna arrays by using differential evolution. IEEE Transactions on Antennas and Propagation.2010; 58(8): 25532561. DOI: doi:10.1109/TAP.2010.2048864 .
[11] Recioui Abdelmadjid, Arhab Nabil, Zeghad Imadeddine. Pattern Design of 2D Antenna Arrays using Biogeography Based Optimization. International Journal of Computer Science, Communication \& Information Technology (CSCIT).2019;6:38-43.

[12] Elizarraras Omar, Mendez Aldo, Reyna Alberto, Panduro Marco A. Design of spherical antenna arrays for a 3D scannable pattern using differential evolution. In: proceedings of Loughborough Antennas \& Propagation Conference (LAPC), Loughborough, United Kingdom, pp. $1-4,2016$. DOI:10.1109/LAPC.2016.7807588

[13] Garza Jesus, Panduro Marco A., Reyna Alberto, Romero Gerardo, del Rio Carlos. Design of UAVs-Based 3D Antenna Arrays for a Maximum Performance in Terms of Directivity and SLL. International Journal of Antennas and Propagation. 2016. DOI :http://dx.doi.org/10.1155/2016/2621862.

[14] Yan-Qiu Z., Zong P. Three-dimensional phased array antenna analysis and simulation. In: Proceedings of 3rd IEEE Int. Symposium Microw. Antenna Propag. and EMC Technol. Wirel. Commun. Beijing, 2009, pp. 538-542.

[15] Vesa Andy, Alexa Florin, Baltă Horia. Comparisons between 2D and 3D Uniform Array Antennas. In: Proceedings of the Federated Conference on Computer Science and Information Systems, Timişoara, Romania. 2015; 5(2): 12851290. DOI: 10.15439/2015F266.

[16] Rogério B. Francisco, M. Fernanda P. , Ana Maria A. C. Rocha Costa. Experiments with Firefly Algorithm", International Conference on Computational Science and Its Applications. Portugal .2014. DOI: https://doi.org/10.1007/9783-319-09129-7_17.

[17] Kennedy J. and Eberhart R. Particle swarm optimization. Proc. IEEE Int. conf. Neural Networks. 1995; pp.1942-1948. DOI: 10.1109/ICNN.1995.488968.

[18] Sharaqa A. and Dib N. Design of linear and elliptical antenna arrays using biogeography based optimization. Arabian Journal for Science and Engineering. 2013; 39(4):2929-2939. DOI: https://doi.org/10.1007/s13369-013-0794-8.

[19] Xin-She Yang,Xingshi He. Firefly Algorithm: Recent Advances and Applications. Int. J. Swarm Intelligence. 2013;1(1): 36-50. DOI: 10.1504/IJSI.2013.055801.

[20] Weng Wei-Chung, Yang Fan, Elsherbeni Atef. Electromagnetics and Antenna Optimization Using Taguchi's Method. Morgan and Claypool Publishers.2007.

10.2200/S00083ED1V01Y200710CEM018.

[21] Yang X-S. Firefly algorithms for multimodal optimization. Watanabe $\mathrm{O}$, Zeugmann $\mathrm{T}$ In: Stochastic algorithms: foundations and applications, SAGA, LNCS. Springer-Verlag. 2009; 5792: 169-178..

[22] Mohammad Asif Zaman, Md. Abdul Matin. Nonuniformly Spaced Linear Antenna Array Design Using Firefly Algorithm. International 
Journal of Microwave Science and Technology. 2012, Article ID 256759, pp.1-8.

[23] Yang X. S. Firefly algorithm, stochastic test functions and design optimization. Int. J. BioInspired Computation. 2010; 2(2):78-84. DOI: 10.1504/IJBIC.2010.032124.

[24] Khan S.U., Qureshi I.M., Zaman F., Basit A., Khan W. Application of Firefly Algorithm to Fault Finding in Linear Arrays Antenna. World Applied Sciences Journal. 2013; 26 (2): 232-238. DOI: 10.5829/idosi.wasj.2013.26.02.1387.

[25] Yang, X. S. Metaheuristic optimization: algorithm analysis and open problems. Proceedings of 10th International Symposium on Experimental Algorithms (SEA 2011) (Eds. P. M. Pardalos and S. Rebennack), Kolimpari, Chania, Greece, May 5-7. Lecture Notes in Computer Sciences. 2011; 6630: 21-32.

[26] Can Umit, Alatas Bilal. Physics Based Metaheuristic Algorithms for Global Optimization. American Journal of Information Science and Computer Engineering, Turkey. 2015; 1(3): 94106.

[27] IztokFister Jr., Xin-She Yang, IztokFister, Janez Brest, Du`san Fister. A Brief Review of Nature-Inspired Algorithms for Optimization. ELEKTROTEHNI “ SKI VESTNIK. 2013; 80(3): 17.

[28] K. R. Subhashini, A. T. Praveen Kumar. Comparative Analysis of Linear and Nonlinear Pattern Synthesis of Hemispherical Antenna Array Using Adaptive Evolutionary Techniques. International Journal of Antennas and Propagation, Department of Electrical Engineering, National Institute of Technology, Rourkela 769008, India. 2014, Article ID 987140: $1-10$. 